\title{
Eustachian tube symptoms are frequent in chronic rhinosinusitis and respond well to endoscopic sinus surgery*
}

\author{
Anastasios Maniakas ${ }^{1,2}$, Martin Desrosiers ${ }^{1,2}$, Marc-Henri Asmar', \\ Mohammad Al Falasi', Leandra Mfuna Endam', Claire Hopkins ${ }^{5}$, \\ Carl Philpott ${ }^{6}$, Sally Erskine ${ }^{6}$, Rupert Smith ${ }^{6}$, Shaun Kilty ${ }^{3}$ \\ Rhinology 56; 2: 118-121, 2018 \\ https://doi.org/10.4193/Rhin 17.165 \\ *Received for publication: \\ July 26, 2017 \\ Accepted: August 18, 2017 \\ ' Centre de Recherche du Centre Hospitalier de I'Université de Montréal (CRCHUM), Montreal, QC, Canada \\ 2 Division of Otolaryngology-Head \& Neck Surgery, Centre Hospitalier de I'Université de Montréal (CHUM), Montreal, QC, Canada \\ ${ }^{3}$ Department of Otolaryngology-Head and Neck Surgery, The University of Ottawa, Ottawa, ON, Canada \\ ${ }^{4}$ Department of Otolaryngology-Head and Neck Surgery, Tawam Hospital, United Arab Emirates \\ ${ }^{5}$ Department of Ear, Nose \& Throat, Guy's and St Thomas' Hospital, London, United Kingdom \\ ${ }^{6}$ Norwich Medical School, University of East Anglia, Norwich, United Kingdom
}

\begin{abstract}
Background: Symptoms of Eustachian tube (ET) dysfunction are seldom assessed in patients with chronic rhinosinusitis (CRS). The Sino-Nasal Outcome Test (SNOT-22) quality-of-life tool includes two questions that specifically screen for symptoms of ET dysfunction ('Ear Fullness';'Ear Pain').
\end{abstract}

Objective: The purpose of this study was to determine the extent to which these ET symptoms were present in patients with CRS, and whether these symptoms respond to endoscopic sinus surgery (ESS).

Methodology: SNOT-22 data collected prospectively at time of recruitment into IRB-approved clinical trials or case-control studies in CRS was pooled to provide a cross section of the frequency and severity of ET dysfunction. When applicable to the trials, the SNOT-22 was repeated at least 3 months following ESS.

Results: Five trials rendering 131 patients were available for assessment. The control group comprised of 251 participants from two case-control studies. 'Ear Fullness' of $\geq 1$ was reported in 80/131 CRS patients compared to 45/251 control patients. 'Ear Pain' of $\geq 1$ was reported in 39/131 CRS patients compared to 33/251 control patients. Following ESS, mean 'Ear Fullness' and 'Ear Pain' scores decreased to 1.17 and 0.73 , respectively.

Conclusion: Symptoms suggestive of ET dysfunction are frequent in CRS, and for most patients the symptoms will decrease postESS to a level comparable with a non-CRS population. Patients' whose ET symptoms do not respond to ESS may represent a target population for emerging therapeutic options for ET dysfunction.

Key words: chronic rhinosinusitis, Eustachian tube dysfunction, endoscopic sinus surgery, SNOT-22, prospective

\section{Introduction}

The Eustachian tube (ET) provides the physiological functions of middle ear pressure equalization, protection, and clearance. ET dysfunction can be divided into two categories: functional defined as the inability to actively dilate the tube; or mechanical - defined as secondary to inflamed mucosa, middle ear disease, hypertrophic adenoids, nasopharyngeal neoplasm, and/or polyps. Tubal integrity and susceptibility to dysfunction are also known to be influenced by allergy and sinonasal disease, especially when these disorders are chronic. Chronic rhinosinusitis (CRS) is one of the most common inflammatory diseases of the nose and paranasal sinuses affecting up to $10 \%$ of the popula- 
tion $^{(1,2)}$. The Sino-Nasal Outcome Test (SNOT-22) is a self-reported symptom-based rhinosinusitis outcome measure tool that is widely accepted and validated for patients with $\mathrm{CRS}^{(3)}$. Patients complete the questionnaire by grading their symptom severity from 0 (not a problem) to 5 (problem as bad as it can be). The SNOT-22 includes two questions related to ET dysfunction, that of "ear fullness" and "ear pain". These symptoms can be quite debilitating to patients ${ }^{(4)}$, leading to increased absenteeism from work or hindered social interactions, and thus deserve further investigation. There have been reports that rhinosinusitis and ET dysfunction can be associated and that endoscopic sinus surgery (ESS) can alleviate such symptoms ${ }^{(5)}$, although it remains to be thoroughly studied.

The aims of this study were to determine the prevalence and severity of ET dysfunction-associated symptoms in CRS patients compared to a control group without known otologic disease or CRS, and to evaluate these symptoms in CRS patients following ESS.

\section{Materials and methods}

This study was approved by the Ethics Review Board of the University of Montreal Health Center (Centre Hospitalier de I'Université de Montréal); CHUM 14.140.

\section{Chronic rhinosinusitis patients}

SNOT-22 data from five institutional review-board-approved prospective clinical trials including both CRS patients with and without nasal polyposis were collected and evaluated. Patients had to have had at least a SNOT-22 completed on the day of surgery to be eligible for this study, while post-ESS SNOT-22 scores were collected from the same patients, when available, at least 3 months following ESS. All patients included in these clinical trials were considered patients at 'high-risk' of CRS recurrence according to our group's previously described criteria ${ }^{(6)}$.

\section{Control group}

Control group SNOT-22 data were retrieved from the Chronic Rhinosinusitis Epidemiology Study (CRES) ${ }^{(7,8)}$ and the ongoing Socioeconomic Cost of Chronic Rhinosinusitis Study (SocCoR) ${ }^{(9)}$ database originating in the United Kingdom. Family and friends of patients attending otolaryngology outpatient clinics and hospital and university staff were recruited as controls. This non-CRS population had no self-reported otologic or nasal disease, active treatment for chronic conditions, nor any hospital admissions in the preceding 12 months.

\section{Statistical analyses}

All data were tabulated using Microsoft Excel and all statistical analyses were performed using STATA 13.1 (STATACorp LP, College Station, TX, USA). Absolute and relative frequencies are presented for categorical and ordinal variables. A two-tailed
Table 1. Demographics and SNOT-22 findings.

\begin{tabular}{|c|c|c|c|}
\hline & $\begin{array}{l}\text { CRS group } \\
(\mathrm{N}=131)\end{array}$ & $\begin{array}{l}\text { Control group } \\
(\mathrm{N}=251)\end{array}$ & P value \\
\hline Age (range) & $52.7(21-86)$ & $47.5(19-80)$ & \\
\hline Female & 52 & 143 & \multirow{2}{*}{$<0.001$} \\
\hline Male & 79 & 96 & \\
\hline SNOT-22 (SD) & $46.4(20.6)$ & 12 (13.6) & $<0.001$ \\
\hline $\begin{array}{l}\text { No. patients with Ear full- } \\
\text { ness } \geq 1(\%)\end{array}$ & 80 (61.1\%) & 45 (17.9\%) & $<0.001$ \\
\hline $\begin{array}{l}\text { No. patients with Ear pain } \\
\geq 1(\%)\end{array}$ & 39 (29.8\%) & 33 (13.2\%) & $<0.001$ \\
\hline $\begin{array}{l}\text { Mean Ear fullness symptom } \\
\text { score if Ear fullness } \geq 1 \text { (SD) }\end{array}$ & $3.08(1.25)$ & $1.84(0.79)$ & $<0.001$ \\
\hline $\begin{array}{l}\text { Mean Ear pain symptom } \\
\text { score if Ear pain } \geq 1 \text { (SD) }\end{array}$ & $2.31(0.95)$ & $1.82(0.5)$ & 0.042 \\
\hline
\end{tabular}

Pearson Chi-square or Fisher's exact test was used to compare the prevalence and proportion of ET dysfunction symptoms between CRS and control groups. Comparison of symptom severity and overall SNOT-22 scores between groups and before and after ESS were evaluated using a two-sample Student T-test with unequal variances. For all statistical analyses, a $\mathrm{p}<0.05$ was considered statistically significant.

\section{Results}

Demographics

A total of 131 CRS patients were included in the CRS group and completed a SNOT-22 on the day of surgery as well as 3 to 4 months post-ESS (mean 3.8 months). The control group comprised of 251 participants having completed the SNOT-22 questionnaire on a single occasion.

\section{SNOT-22 according to ear symptom in CRS patients}

The mean SNOT-22 score for CRS patients with a score of $\geq 1$ for 'ear fullness' was significantly higher than in CRS patients with a score of 0 (53.2 vs. 35.8; $p<0.001$ ). Similarly, CRS patients with a score of $\geq 1$ for 'ear pain' had a significantly higher overall SNOT-22 score than CRS patients with a score of 0 (63.4 vs. 39.2; $\mathrm{p}<0.001)$. Nasal polyposis (NP) $(92 \%$ CRS with NP; $8 \%$ without $\mathrm{NP}$ ) was not associated with higher 'ear pain', 'ear fullness', or overall SNOT-22 scores ( $p=0.886 ; p=0.660 ; p=0.600$, respectively)

\section{Evolution of 'ear fullness'}

Of the 80 CRS patients with a score of $\geq 1$ for 'ear fullness', 66 $(82.5 \%)$ completed a SNOT-22 score between 3 and 4 months post-ESS (mean 3.8 months). Mean ear fullness score statistically significantly decreased post-ESS to $1.17(p<0.001)$ (Figure 1), while $78.8 \%$ (52 of 66 ) reported an improvement in their 'ear fullness' score (mean improvement $=2.5$; range 1 to 5 ). Five patients 


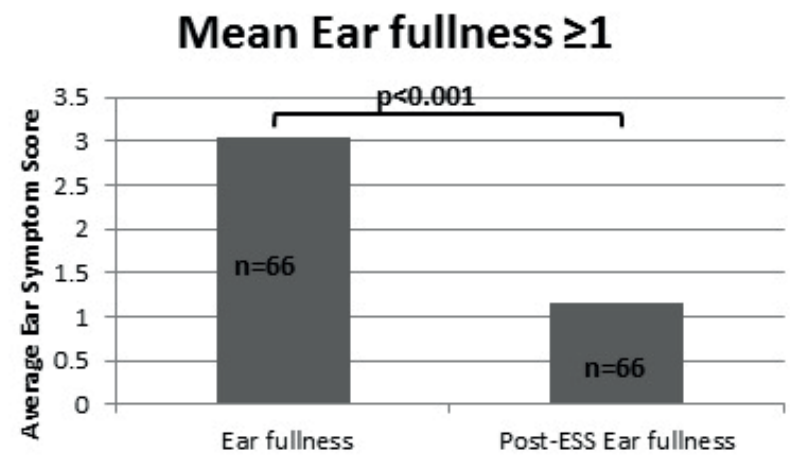

Figure 1. Evolution of mean ear fullness score following endoscopic sinus surgery.

\section{Mean Ear pain $\geq 1$}

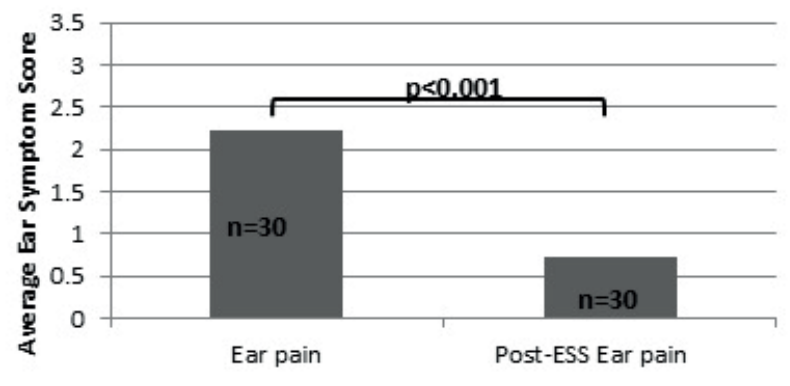

Figure 2. Evolution of mean ear pain score following endoscopic sinus surgery.

(7.6\%) reported symptom deterioration.

\section{Evolution of 'ear pain'}

Of the 39 CRS patients with a score of $\geq 1$ for 'ear pain', 30 (76.9\%) completed a SNOT-22 score between 3 and 4 months post-ESS (mean 3.8 months). Mean ear pain score statistically significantly decreased post-ESS to $0.73(p<0.001)$ (Figure 2$)$, while $73.3 \%$ (22 of 30) reported an improvement in their 'ear fullness' score (mean improvement $=2.2$; range 1 to 3 ). Three patients (10\%) reported symptom deterioration.

\section{Discussion}

The current literature on ET dysfunction symptoms in patients with CRS is very limited. To our knowledge, this is the first study where the effect of ESS for CRS on ET dysfunction symptoms are prospectively evaluated and compared to a control group. Our findings reveal that symptoms suggestive of ET dysfunction are quite frequent in patients with CRS who fail maximal medical therapy and are proceeding to ESS. More specifically, 'ear fullness' was a reported symptom in close to two thirds of patients, while 'ear pain' was reported in one third of CRS patients. These rates are significantly higher than Stoikes et al.'s ${ }^{(5)}$ previously published findings ( $42 \%$ and $15 \%$, respectively). Furthermore, when present, 'ear fullness' and 'ear pain' were both significantly more prevalent and more severe in CRS patients compared to the control group. Overall, it is clear that the prevalence of ET dysfunction in CRS has been greatly underappreciated, particularly in patients with severe disease.

Our second significant finding is the important treatment effect CRS patients had following ESS. Approximately $75 \%$ of reported 'ear fullness' and 'ear pain' had a favourable evolution post-ESS, with a mean improvement of more than 2 points on 5 . These findings are slightly inferior to Stoikes et al.'s ${ }^{(5)}$ findings $(84.3 \%$ and $84 \%$, respectively), although one must consider the important recall bias and variability of post-ESS response timeframe of their retrospective study. Others have demonstrated the improvement of ear-associated SNOT-22 symptoms without however providing a detailed categorization ${ }^{(10-12)}$. Our findings do show that for the majority of patients with ET symptoms associated with CRS, these improve following ESS. It remains to be determined why these symptoms do not improve for a small proportion of patients and if these are patients with ET dysfunction that requires additional otology specific therapy.

\section{Limitations}

The proportion of females was significantly higher in the control group, and as it has been described in the literature, females tend to report higher SNOT-22 scores ${ }^{(7)}$. This can unfortunately hinder our group comparability. However, when males and females average SNOT-22 scores were compared within each group, both CRS and control group females had proportionally higher scores. Overall, the effect of this inter-group difference can only lead to an underestimation of the symptoms in the CRS group, which in turn strengthens the already significant findings we have reported. Additionally, although we demonstrated no difference between CRS populations with or without polyposis, the proportion of patients without polyposis was significantly smaller than the former rendering it a slightly underpowered analysis. Further, our follow-up period was short and we therefore were unable to assess the long-term effect of ESS on ET dysfunction-associated symptoms. Finally, an objective measure of ET function such as tympanometry would help identify patients with primary ET pathology.

\section{Future directions}

Although this prospective study on ET dysfunction-associated symptoms demonstrates the effect CRS can have on such symptoms, a more thorough evaluation of ET dysfunction with the Eustachian Tube Dysfunction Questionnaire (ETDQ-7) ${ }^{(13)}$ would be most appropriate. Furthermore, additional audiometric tools such as an audiogram and/or tympanometry may be used to complement patient evaluation. What remains to be addressed is the management of CRS patients with persistent and debilitating ET dysfunction symptoms, even following ESS where there remains a prevalence of $7.6 \%$ for ear fullness and 
$10 \%$ for ear pain. Alternative management is available and should be considered; one of these may be the novel surgical technique of ET balloon dilatation that has been shown to be a possible therapeutic option ${ }^{(14)}$ but remains to be validated with randomized controlled trials and long-term follow-up studies.

\section{Conclusion}

Symptoms suggesting ET dysfunction have been underestimated in the CRS population, especially in patients with severe disease. Our findings depict the substantial prevalence of 'ear fullness' and 'ear pain' in patients undergoing ESS for CRS, compared to a control population, while also demonstrating the strong positive treatment effect of ESS on these symptoms. We suggest that further study into ET dysfunction in CRS is needed to better understand the origin of these symptoms and to evaluate ideal treatment options for patients whose symptoms do not respond to surgical treatment of CRS.

\section{Acknowledgement}

This material has never been published and is not currently under evaluation in any other peer-reviewed publication. The first author has received funding through the Canadian Institutes of Health Research (CIHR) \& the Fonds de Recherche du QuébecSanté (FRQS).

\section{Authorship contribution}

AM: Study design; Data collection and analysis; Manuscript drafting, reviewing, final approval, accountability for all aspects of the work; MD: Study design; Data analysis; Manuscript writing, reviewing, final approval, accountability for all aspects of the work; MMA: Data collection and analysis; Manuscript reviewing, final approval, accountability for all aspects of the work; MAF: Data analysis; Manuscript drafting, final approval, accountability for all aspects of the work; LME: Data collection and analysis; Manuscript drafting; final approval, accountability for all aspects of the work; $\mathrm{CH}$ : Data analysis; Manuscript drafting; final approval, accountability for all aspects of the work; CP: Data analysis; Manuscript drafting, reviewing, final approval, accountability for all aspects of the work; SE: Data collection and analysis; Manuscript drafting; final approval, accountability for all aspects of the work; RS: Data collection and analysis; Manuscript drafting; final approval, accountability for all aspects of the work; SK: Study Design; Data collection and analysis; Manuscript drafting, reviewing, final approval, accountability for all aspects of the work.

\section{Conflict of interest}

No conflict of interest.

\section{References}

1. Hastan D, Fokkens WJ, Bachert C, et al Chronic rhinosinusitis in Europe--an underestimated disease. A GA(2)LEN study. Allergy. 2011;66(9):1216-1223.

2. Chen Y, Dales R, Lin M. The epidemiology of chronic rhinosinusitis in Canadians. Laryngoscope. 2003;113(7):1199-1205.

3. Hopkins C, Gillett S, Slack R, Lund VJ, Browne JP. Psychometric validity of the 22-item Sinonasal Outcome Test. Clin Otolaryngol. 2009 Oct;34(5):447-54.

4. Bakhshaee M, Ardakani HP, Ghazizadeh AH, Movahed R, Jarahi L, Rajati M. Middle ear function in sinonasal polyposis. Eur Arch Otorhinolaryngol. 2016 Oct;273(10):2911-6.

5. Stoikes NF, Dutton JM. The effect of endoscopic sinus surgery on symptoms of eustachian tube dysfunction. Am J Rhinol. 2005;19(2):199-202.

6. Maniakas A, Desrosiers M. Azithromycin add-on therapy in high-risk postendoscopic sinus surgery patients failing corticosteroid irrigations: A clinical practice audit. Am J Rhinol Allergy. 2014 Mar-Apr;28(2):151-5.

7. Erskine SE, Hopkins C, Clark A, et al. SNOT-22 in a control population.Clin Otolaryngol. 2017 Feb;42(1):81-85.

8. Philpott C, Erskine S, Hopkins C, et al. A case-control study of medical, psychological and socio-economic factors influencing the severity of chronic rhinosinusitis. Rhinology. 2016;54(2):134-140.

9. Smith R, Erskine S, Philpott C. Preliminary results of a questionnaire based study to identify the socio-economic costs of Chronic Rhinosinusitis (CRS) to patients and society. 2016; http://www.britishrhinologicalsociety.org.uk/meetings/abstractdisplay. php?ID=161.

10. Kennedy JL, Hubbard MA, Huyett P, Patrie JT, Borish L, Payne SC. Sino-nasal outcome test (SNOT-22): a predictor of postsurgical improvement in patients with chronic sinusitis. Ann Allergy Asthma Immunol. 2013;111(4):246-251.e242.

11. Yu VM, Yu JM. Improvement in Eustachian Tube Function after Endoscopic Sinus Surgery for Polypoid Chronic Rhinosinusitis (Abstract). Otolaryngology - Head \& Neck Surgery. 2013.

12. DeConde AS, Mace JC, Bodner T, et al. SNOT-22 quality of life domains differentially predict treatment modality selection in chronic rhinosinusitis. Int Forum Allergy Rhinol. 2014 Dec;4(12):972-9.

13. McCoul ED, Anand VK, Christos PJ. Validating the clinical assessment of eus- tachian tube dysfunction: The Eustachian Tube Dysfunction Questionnaire (ETDQ-7). Laryngoscope. 2012;122(5):1137-1141.

14. Williams B, Taylor BA, Clifton N, Bance M. Balloon dilation of the Eustachian tube: a tympanometric outcomes analysis. J Otolaryngol Head Neck Surg. 2016;45:13.

Shaun Kilty, MD, FRCSC

Department of Otolaryngology

Head and Neck Surgery

The Ottawa Hospital

University of Ottawa

259-737 Parkdale Ave.

Ottawa

Ontario K1Y 1 J8

Canada

Tel: +1-(613) 798-5555 ext. 18514

Fax: +1-(613) 729-2412

E-mail: kiltysj@gmail.com 